PROCEEDINGS OF THE

AMERICAN MATHEMATICAL SOCIETY

Volume 129, Number 8, Pages 2343-2351

S 0002-9939(00)05844-5

Article electronically published on December 28, 2000

\title{
A FORMULA FOR $k$-HYPONORMALITY OF BACKSTEP EXTENSIONS OF SUBNORMAL WEIGHTED SHIFTS
}

\author{
IL BONG JUNG AND CHUNJI LI
}

(Communicated by David R. Larson)

\begin{abstract}
Let $\alpha: \alpha_{0}, \alpha_{1}, \cdots$ be a weight sequence of positive real numbers and let $W_{\alpha}$ be a subnormal weighted shift with a weight sequence $\alpha$. Consider an extended weight sequence $\alpha(x): x, \alpha_{0}, \alpha_{1}, \cdots$ with $0<x \leq \alpha_{0}$ and let $H E(\alpha, k):=\left\{x>0: W_{\alpha(x)}\right.$ is $k$-hyponormal $\}$ for $k \in \mathbb{N} \cup\{\infty\}$, where $\mathbb{N}$ is the set of natural numbers. We obtain a formula to find the interval $H E(\alpha, k) \backslash H E(\alpha, k+1)$, which provides several examples to distinguish the classes of $k$-hyponormal operators from one another.
\end{abstract}

\section{INTRODUCTION AND PRELIMINARIES}

Let $\mathcal{H}$ be a separable, infinite dimensional, complex Hilbert space and let $\mathcal{L}(\mathcal{H})$ denote the algebra of all bounded linear operators on $\mathcal{H}$. For $A, B \in \mathcal{L}(\mathcal{H})$, let $[A, B]=A B-B A$. We say that an $n$-tuple $T=\left(T_{1}, \cdots, T_{n}\right)$ of operators in $\mathcal{L}(\mathcal{H})$ is hyponormal if the operator matrix $\left(\left[T_{j}^{*}, T_{i}\right]\right)_{i, j=1}^{n}$ is positive on the direct sum of $n$ copies of $\mathcal{H}$. For arbitrary positive integer $k, T \in \mathcal{L}(\mathcal{H})$ is $k$-hyponormal if $\left(I, T, \cdots, T^{k}\right)$ is hyponormal. It is well known that $T$ is subnormal if and only if $T$ is $\infty$-hyponormal (cf. [Br], $\mathrm{Hal}]$ ).

Let $\alpha: \alpha_{0}, \alpha_{1}, \cdots$ be a sequence of positive real numbers. Let $x>0$ and let $\alpha(x): x, \alpha_{0}, \alpha_{1}, \cdots$ be an augmented weight sequence. For $k \in \mathbb{N} \cup\{\infty\}$, we write $H E(\alpha, k)$ for the set of all positive real variable $x$ such that $W_{\alpha(x)}$ is $k$ hyponormal (cf. [CuF2]). It follows from [Cu1] that if $\alpha(x): x, \sqrt{\frac{2}{3}}, \sqrt{\frac{3}{4}}, \sqrt{\frac{4}{5}}, \cdots$, then there exists a sequence $\left\{\lambda_{k}\right\}_{k=1}^{\infty}$ of positive numbers with $\lim _{k \rightarrow \infty} \lambda_{k}=\sqrt{\frac{1}{2}}$ such that $\lambda_{k}>\lambda_{k+1}(k \geq 1)$ and $\operatorname{HE}(\alpha, k)=\left(0, \lambda_{k}\right]$, where $\lambda_{1}=\sqrt{\frac{2}{3}}, \lambda_{2}=$ $\frac{3}{4}, \lambda_{3}=\sqrt{\frac{8}{15}}, \lambda_{4}=\sqrt{\frac{25}{48}}, \cdots$ and $H E(\alpha, \infty)=\left(0, \sqrt{\frac{1}{2}}\right]$, which gives an example that distinguishes the classes of $k$-hyponormal operators from one another. In this paper, we obtain a formula that captures such examples.

Received by the editors January 22, 1999 and, in revised form, December 7, 1999.

2000 Mathematics Subject Classification. Primary 47B37.

Key words and phrases. Subnormal weighted shifts, $k$-hyponormal weighted shifts.

The first author was partially supported by KOSEF grant 971-0102-006-2 and the Korea Research Foundation made in the program year of 1998, 1998-015-D00019. The second author was partially supported by TGRC-KOSEF. 
Note that for a unilateral weighted shift $W_{\alpha}$ with $\alpha_{n}=\alpha_{n+1}$ for some $n \in$ $\mathbb{N} \cup\{0\}, 2$-hyponormality immediately forces the weight sequence $\alpha$ to be flat, that is, $\alpha_{1}=\alpha_{2}=\cdots$ (cf. [Cu1]). In Sta], J. Stampfli had previously established this for subnormal shifts, so if the subnormal weighted shift is not flat, its weight sequence $\alpha$ satisfies $\alpha_{0}<\alpha_{1}<\alpha_{2}<\cdots<\alpha_{n}<\cdots$. Throughout this paper we may assume that the subnormal weighted shift $W_{\alpha}$ satisfies $\alpha_{0}<\alpha_{1}<\alpha_{2}<\cdots<\alpha_{n}<\cdots$ to escape the trivial case.

A weighted shift $W_{\alpha}$ is said to be recursively generated if there exist an integer $r \geq 1$ and a vector $\psi=\left(\psi_{0}, \cdots, \psi_{r-1}\right) \in \mathbb{C}^{r}$ such that $\gamma_{n}=\psi_{r-1} \gamma_{n-1}+\cdots+$ $\psi_{0} \gamma_{n-r}(n \geq r)$, where $\gamma_{n}(n \geq 0)$ is the moment of $W_{\alpha}$, i.e., $\gamma_{0}:=1, \gamma_{n}:=\alpha_{0}^{2} \cdots \alpha_{n-1}^{2}$ $(n \geq 1)$, equivalently,

$$
\alpha_{n}^{2}=\psi_{r-1}+\frac{\psi_{r-2}}{\alpha_{n-1}^{2}}+\cdots+\frac{\psi_{0}}{\alpha_{n-1}^{2} \cdots \alpha_{n-r+1}^{2}} \quad(n \geq r) .
$$

The smallest such integer $r$ is called the rank of $\gamma$. A weighted shift $W_{\alpha}$ is nonrecursively generated if it is not recursively generated. Note that a subnormal weighted shift is recursively generated if and only if the corresponding probability measure has finite support (cf. [ShT, p. 6] or [CuF1]).

For the moment sequence $\left\{\gamma_{n}\right\}_{n=0}^{\infty}$ of $W_{\alpha}$, we denote

$$
A(i, j):=\left[\begin{array}{cccc}
\gamma_{i} & \gamma_{i+1} & \cdots & \gamma_{i+j} \\
\gamma_{i+1} & \gamma_{i+2} & \cdots & \gamma_{i+j+1} \\
\vdots & \vdots & \ddots & \vdots \\
\gamma_{i+j} & \gamma_{i+j+1} & \cdots & \gamma_{i+2 j}
\end{array}\right]
$$

If a subnormal weighted shift $W_{\alpha}$ is recursively generated and rank $\gamma=r$, then $\operatorname{det} A(i, r-1) \neq 0$ and $\operatorname{det} A(i, j)=0$ for any $i \geq 1, j \geq r$. Note that if a subnormal weighted shift $W_{\alpha}$ is non-recursively generated, then $\operatorname{det} A(i, j)>0$ for any positive integers $i$ and $j$ (cf. $\mathrm{CuF} 3$ ).

\section{A FORMULA FOR $k$-HYPONORMALITY}

2.1. Non-recursively generated type. Let $\alpha: \alpha_{0}<\alpha_{1}<\alpha_{2}<\cdots<\alpha_{n}<\cdots$ be a sequence of positive real numbers. Let $x>0$ and let $\alpha(x): x, \alpha_{0}, \alpha_{1}, \cdots$ be an augmented weight sequence. Assume that $W_{\alpha}$ is a non-recursively generated subnormal weighted shift. For brevity, let us put $t:=\frac{1}{x^{2}}$. Then it follows from [Cu1, Theorem 4] that $W_{\alpha(x)}$ is $k$-hyponormal if and only if

$$
D_{k}(t):=\left[\begin{array}{ccccc}
t & \gamma_{0} & \gamma_{1} & \ldots & \gamma_{k-1} \\
\gamma_{0} & \gamma_{1} & \gamma_{2} & \cdots & \gamma_{k} \\
\vdots & \vdots & \vdots & \ddots & \vdots \\
\gamma_{k-1} & \gamma_{k} & \gamma_{k+1} & \cdots & \gamma_{2 k-1}
\end{array}\right]
$$

is non-negative, where $\gamma_{0}:=1, \gamma_{n+1}:=\alpha_{n}^{2} \gamma_{n}(n \geq 0)$. Note that $d_{k}(t):=\operatorname{det} D_{k}(t)$ is a polynomial in $t$ of degree 1 . Since $W_{\alpha}$ is non-recursively generated subnormal, the coefficient of $t$ in $d_{k}(t)$, $\operatorname{det} A(1, k-1)$, is positive. Hence $d_{k}(t)$ has a unique zero. We write $t_{k}:=t_{k}(\alpha)$ for the unique zero of $d_{k}(t)$. 
Theorem 2.1. Let $\alpha: \alpha_{0}<\alpha_{1}<\alpha_{2}<\cdots<\alpha_{n}<\cdots$ be a sequence of positive real numbers. Assume that $W_{\alpha}$ is a non-recursively generated subnormal weighted shift. Let $x>0$ and let $\alpha(x): x, \alpha_{0}, \alpha_{1}, \cdots$ be the associated augmented weight sequence. Let $t_{k}:=t_{k}(\alpha)$ be the unique zero of $\operatorname{det} D_{k}(t)$, where $t:=\frac{1}{x^{2}}$. Then

$$
t_{k+1}(\alpha)=t_{k}(\alpha)+\frac{[\operatorname{det} A(0, k)]^{2}}{\operatorname{det} A(1, k-1) \cdot \operatorname{det} A(1, k)}
$$

for all $k=1,2, \cdots$.

For an $n \times n$ matrix $A=\left[a_{i j}\right]_{1 \leq i, j \leq n}$, we write

$$
A\left(\begin{array}{cccc}
i_{1} & i_{2} & \cdots & i_{p} \\
k_{1} & k_{2} & \cdots & k_{p}
\end{array}\right)=\left|\begin{array}{cccc}
a_{i_{1} k_{1}} & a_{i_{1} k_{2}} & \cdots & a_{i_{1} k_{p}} \\
a_{i_{2} k_{1}} & a_{i_{2} k_{2}} & \cdots & a_{i_{2} k_{p}} \\
\vdots & \vdots & \ddots & \vdots \\
a_{i_{p} k_{1}} & a_{i_{p} k_{2}} & \cdots & a_{i_{p} k_{p}}
\end{array}\right|
$$

for a minor of $A$ of order $p$. We recall a fundamental result from [Gan, p. 22] as follows.

Lemma 2.2. Let $A=\left[a_{i j}\right]_{1 \leq i, j \leq n}$ be an $n \times n$ matrix. Then

$$
\begin{gathered}
\operatorname{det} A=\sum_{1 \leq i_{1}<i_{2}<\cdots<i_{p} \leq n} A\left(\begin{array}{cccc}
k_{1} & k_{2} & \cdots & k_{p} \\
i_{1} & i_{2} & \cdots & i_{p}
\end{array}\right) \cdot(-1)^{\sum_{v=1}^{p} i_{v}+\sum_{v=1}^{p} k_{v}} \\
\cdot A\left(\begin{array}{cccc}
k_{1}^{\prime} & k_{2}^{\prime} & \cdots & k_{n-p}^{\prime} \\
i_{1}^{\prime} & i_{2}^{\prime} & \cdots & i_{n-p}^{\prime}
\end{array}\right)
\end{gathered}
$$

where $i_{1}<i_{2}<\cdots<i_{p}$ and $i_{1}^{\prime}<i_{2}^{\prime}<\cdots<i_{n-p}^{\prime}$ form a complete system of indices $1,2, \cdots, n$, as do $k_{1}<k_{2}<\cdots<k_{p}$ and $k_{1}^{\prime}<k_{2}^{\prime}<\cdots<k_{n-p}^{\prime}$.

Proof of Theorem 2.1. Let $M_{k}^{(i)}$ be the $k \times k$ matrix obtained by removing the $(i+1)$-th column from the matrix

$$
\left[\begin{array}{ccccc}
\gamma_{0} & \gamma_{1} & \gamma_{2} & \cdots & \gamma_{k} \\
\gamma_{1} & \gamma_{2} & \gamma_{3} & \cdots & \gamma_{k+1} \\
\vdots & \vdots & \vdots & & \vdots \\
\gamma_{k-1} & \gamma_{k} & \gamma_{k+1} & \cdots & \gamma_{2 k-1}
\end{array}\right]
$$

and let $d_{k}^{(i)}:=\operatorname{det} M_{k}^{(i)}$ for $i=0,1,2, \cdots, k$. Let us expand $d_{k}(t)=\operatorname{det} D_{k}(t)$ by the first row to obtain

$$
d_{k}(t)=t d_{k}^{(0)}-\gamma_{0} d_{k}^{(1)}+\gamma_{1} d_{k}^{(2)}-\cdots+(-1)^{k} \gamma_{k-1} d_{k}^{(k)}
$$

Let $t_{k}:=t_{k}(\alpha)$ be the zero of $d_{k}(t)$. Since $d_{k}^{(0)}>0$ for $k=1,2, \cdots$, we have

$$
t_{k}=\gamma_{0} \frac{d_{k}^{(1)}}{d_{k}^{(0)}}-\gamma_{1} \frac{d_{k}^{(2)}}{d_{k}^{(0)}}+\cdots+(-1)^{k+1} \gamma_{k-1} \frac{d_{k}^{(k)}}{d_{k}^{(0)}} \quad(k \in \mathbb{N})
$$


Hence

$$
\begin{aligned}
d_{k}^{(0)} d_{k+1}^{(0)}\left(t_{k+1}-t_{k}\right)= & \gamma_{0}\left(d_{k}^{(0)} d_{k+1}^{(1)}-d_{k}^{(1)} d_{k+1}^{(0)}\right)-\gamma_{1}\left(d_{k}^{(0)} d_{k+1}^{(2)}-d_{k}^{(2)} d_{k+1}^{(0)}\right) \\
& +\cdots+(-1)^{k-1} \gamma_{k-1}\left(d_{k}^{(0)} d_{k+1}^{(k)}-d_{k}^{(k)} d_{k+1}^{(0)}\right) \\
& +(-1)^{k} \gamma_{k} d_{k}^{(0)} d_{k+1}^{(k+1)}
\end{aligned}
$$

We first denote

$$
\begin{aligned}
& \widetilde{A}_{(2 k+1) \times(2 k+1)}^{(i)} \\
& :=\left[\begin{array}{cccccc|cc}
\gamma_{0} & \gamma_{1} & \cdots & \gamma_{i+1} & \cdots & \gamma_{k} & & \gamma_{k+1} \\
\gamma_{1} & \gamma_{2} & \cdots & \gamma_{i+2} & \cdots & \gamma_{k+1} & O_{(k+1) \times(k-1)} & \gamma_{k+2} \\
\vdots & \vdots & & \vdots & & \vdots & & \vdots \\
\gamma_{k} & \gamma_{k+1} & \cdots & \gamma_{k+i+1} & \cdots & \gamma_{2 k} & & \gamma_{2 k+1} \\
\hline \gamma_{0} & & & \gamma_{i+1} & & & & \\
\gamma_{1} & O_{k \times i} & & \gamma_{i+2} & O_{k \times(k-i-1)} & & B_{k+1}^{(i+1)} & \\
\vdots & & & \vdots & & &
\end{array}\right],
\end{aligned}
$$

where $O_{i \times j}$ is the $i \times j$ zero matrix and $B_{k+1}^{(i+1)}$ is the $k \times k$ submatrix obtained by removing the $(i+1)$-column from the matrix

$$
\left[\begin{array}{ccc}
\gamma_{1} & \cdots & \gamma_{k+1} \\
\vdots & \ddots & \vdots \\
\gamma_{k} & \cdots & \gamma_{2 k}
\end{array}\right]
$$

$(i=0,1, \cdots, k)$. Let $v(i, j)=\left[\gamma_{i}, \gamma_{i+1}, \cdots, \gamma_{i+j}\right]^{T}$. Since the $k+2$ columns of the submatrix

$$
\left[\begin{array}{cccc}
\gamma_{0} & \cdots & \gamma_{k} & \gamma_{k+1} \\
\vdots & \ddots & \vdots & \vdots \\
\gamma_{k} & \cdots & \gamma_{2 k} & \gamma_{2 k+1}
\end{array}\right]
$$

in the upper half submatrix of the matrix $\widetilde{A}_{(2 k+1) \times(2 k+1)}^{(i)}$ are linearly dependent, there exist real numbers $\phi_{i}, i=0,1, \cdots, k$, such that $v(k+1, k)=\phi_{0} v(0, k)+$ $\cdots+\phi_{k} v(k, k)$, which proves easily that the columns of $\widetilde{A}_{(2 k+1) \times(2 k+1)}^{(i)}$ are linearly dependent. Hence

$$
\operatorname{det} \widetilde{A}_{(2 k+1) \times(2 k+1)}^{(i)}=0 \quad \text { for } \quad i=0,1, \cdots, k \text {. }
$$


For brevity we write $\widetilde{A}:=\widetilde{A}_{(2 k+1) \times(2 k+1)}^{(i)}$. Then, applying Lemma 2.2 (using $\widetilde{A}$ and the last $k$ rows of $\widetilde{A}$ ), we have that

$$
\begin{aligned}
& \operatorname{det} \widetilde{A}=\sum_{1 \leq i_{1}<i_{2}<\cdots<i_{k} \leq 2 k+1} \widetilde{A}\left(\begin{array}{ccc}
k+2 & \cdots & 2 k+1 \\
i_{1} & \cdots & i_{k}
\end{array}\right) \\
& \cdot(-1)^{\sum_{v=1}^{k} i_{v}+\sum_{v=k+2}^{2 k+1} v} \cdot \widetilde{A}\left(\begin{array}{ccc}
1 & \cdots & k+1 \\
i_{1}^{\prime} & \cdots & i_{k+1}^{\prime}
\end{array}\right) \\
& \text { where } i_{1}<i_{2}<\cdots<i_{k} \text { and } i_{1}^{\prime}<i_{2}^{\prime}<\cdots<i_{k+1}^{\prime} \\
& \text { form a complete system of indices } 1,2, \cdots, 2 k+1 \text {, } \\
& =(-1)^{1+\sum_{v=k+2}^{2 k} v+\sum_{v=k+2}^{2 k+1}{ }^{v}} d_{k}^{(i+1)} d_{k+1}^{(0)} \\
& +(-1)^{\sum_{v=k+2}^{2 k+1} v+\sum_{v=k+2}^{2 k+1} v} \operatorname{det} B_{k+1}^{(i+1)} \operatorname{det} A(0, k) \\
& +(-1)^{(i+2)+\sum_{v=k+2}^{2 k} v+\sum_{v=k+2}^{2 k+1} v} \\
& \left|\begin{array}{ccccccc}
\gamma_{i+1} & \gamma_{1} & \cdots & \gamma_{i} & \gamma_{i+2} & \cdots & \gamma_{k} \\
\gamma_{i+2} & \gamma_{2} & \cdots & \gamma_{i+1} & \gamma_{i+3} & \cdots & \gamma_{k+1} \\
\vdots & \vdots & \ddots & \vdots & \vdots & \ddots & \vdots \\
\gamma_{k+i} & \gamma_{k} & \cdots & \gamma_{k+i-1} & \gamma_{k+i+1} & \cdots & \gamma_{2 k-1}
\end{array}\right| \cdot d_{k+1}^{(i+1)} \\
& =d_{k}^{(i+1)} d_{k+1}^{(0)}+\operatorname{det} B_{k+1}^{(i+1)} \operatorname{det} A(0, k) \\
& -(-1)^{(i+2)}\left|\begin{array}{ccccccc}
\gamma_{i+1} & \gamma_{1} & \cdots & \gamma_{i} & \gamma_{i+2} & \cdots & \gamma_{k} \\
\gamma_{i+2} & \gamma_{2} & \cdots & \gamma_{i+1} & \gamma_{i+3} & \cdots & \gamma_{k+1} \\
\vdots & \vdots & \ddots & \vdots & \vdots & \ddots & \vdots \\
\gamma_{k+2} & \gamma_{k} & \cdots & \gamma_{k+i-1} & \gamma_{k+i+1} & \cdots & \gamma_{2 k-1}
\end{array}\right| \cdot d_{k+1}^{(i+1)} \\
& =d_{k}^{(i+1)} d_{k+1}^{(0)}+\operatorname{det} B_{k+1}^{(i+1)} \operatorname{det} A(0, k)-d_{k}^{(0)} d_{k+1}^{(i+1)} .
\end{aligned}
$$

Hence by (2.4) we have

$$
d_{k}^{(0)} d_{k+1}^{(i+1)}-d_{k}^{(i+1)} d_{k+1}^{(0)}=\operatorname{det} A(0, k) \cdot \operatorname{det} B_{k+1}^{(i+1)} \quad(i=0, \cdots, k-1) .
$$

Since $d_{k+1}^{(k+1)}=\operatorname{det} A(0, k)$, by $(2.3)$ and $(2.5)$ we have

$$
\begin{aligned}
d_{k}^{(0)} d_{k+1}^{(0)}\left(t_{k+1}-t_{k}\right) & =[\operatorname{det} A(0, k)] \cdot\left[\sum_{i=0}^{k-1}(-1)^{i} \gamma_{i} \operatorname{det} B_{k+1}^{(i+1)}+(-1)^{k} \gamma_{k} d_{k}^{(0)}\right] \\
& =[\operatorname{det} A(0, k)]^{2}
\end{aligned}
$$

which proves the theorem.

Since $\operatorname{det} A(1, k-1)>0$ for $k=1,2, \cdots, d_{k}(t) \geq 0 \Longleftrightarrow t \geq t_{k}(\alpha)$ for all $k=1,2, \cdots$. Since $\operatorname{det} A(0, k)>0$ for $k=1,2, \cdots$ and $t_{1}(\alpha)=\frac{\gamma_{0}{ }^{2}}{\gamma_{1}}$, by $(2.1)$, $0<t_{1}(\alpha)<t_{2}(\alpha)<\cdots$. Hence we have the following corollary.

Corollary 2.3. Let $\alpha: \alpha_{0}<\alpha_{1}<\alpha_{2}<\cdots<\alpha_{n}<\cdots$ be a sequence of positive real numbers. Let $x>0$ and let $\alpha(x): x, \alpha_{0}, \alpha_{1}, \cdots$ be the associated augmented weight sequence. Assume that $W_{\alpha}$ is a non-recursively subnormal weighted shift. 
Let $t_{k}:=t_{k}(\alpha)$ be the unique zero of $\operatorname{det} D_{k}(t)$, where $t:=\frac{1}{x^{2}}$. Then for any $k \in \mathbb{N}$,

$$
H E(\alpha, k) \backslash H E(\alpha, k+1)=\left(\frac{1}{\sqrt{t_{k+1}(\alpha)}}, \frac{1}{\sqrt{t_{k}(\alpha)}}\right] .
$$

In particular, $H E(\alpha, \infty)=\bigcap_{k=1}^{\infty}\left(0, \frac{1}{\sqrt{t_{k}(\alpha)}}\right]$.

2.2. Recursively generated type. Let $W_{\alpha}$ be a recursively generated subnormal weighted shift and let $\operatorname{rank} \gamma=r$. Then $\operatorname{det} A(1, i)>0$ for $i=1, \cdots, r-1$ and $\operatorname{det} A(1, i)=0$ for $i \geq r$. According to the proof of Theorem 2.1,

$$
t_{k+1}(\alpha)=t_{k}(\alpha)+\frac{[\operatorname{det} A(0, k)]^{2}}{\operatorname{det} A(1, k-1) \cdot \operatorname{det} A(1, k)}
$$

for all $k=1,2, \cdots, r-1$. Since rank $D_{k+1}(t)=\operatorname{rank} D_{k}(t)(k \geq r)$ by [CuF1, we have that $D_{k+1}(t) \geq 0 \Longleftrightarrow D_{k}(t) \geq 0(k \geq r)$. So $t_{k+1}(\alpha)=t_{k}(\alpha)$ for all $k \geq r$. Hence we have the following proposition.

Proposition 2.4. Assume $W_{\alpha}$ is a recursively generated subnormal weighted shift and let rank $\gamma=r$. Let $x>0$ and let $\alpha(x): x, \alpha_{0}, \alpha_{1}, \cdots$ be an augmented weight sequence. Let $t_{k}:=t_{k}(\alpha)$ be the unique zero of $\operatorname{det} D_{k}(t)$, where $t:=\frac{1}{x^{2}}$. Then we have

(i) for $p \leq r-1$,

$$
H E(\alpha, p) \backslash H E(\alpha, p+1)=\left(\frac{1}{\sqrt{t_{p+1}(\alpha)}}, \frac{1}{\sqrt{t_{p}(\alpha)}}\right],
$$

(ii) for any $p \geq r, H E(\alpha, p)=H E(\alpha, \infty)$.

\section{EXAMPLES}

3.1. Non-recursively generated type. Given any non-recursively generated subnormal weighted shift $W_{\alpha}$, by Theorem 2.1 and Corollary 2.3 the one step extension of $W_{\alpha}$ provides several examples to distinguish the classes of $k$-hyponormal operators. For example, we may recapture Curto's example [Cu1, Proposition 7] as follows.

Example 3.1. Let $\alpha_{n}:=\sqrt{\frac{n+2}{n+3}} \quad(n \geq 0)$. It follows from [Cu1] (or Example $3.2)$ that $W_{\alpha(x)}$ is subnormal if and only if $0<x \leq \sqrt{\frac{1}{2}}$. Since the support of Berger measure corresponded by $W_{\alpha}$ is not finite, $W_{\alpha}$ is non-recursively generated. Applying Theorem 2.1, we have $t_{1}=\frac{3}{2}, t_{2}=\frac{16}{9}, t_{3}=\frac{15}{8}, t_{4}=\frac{48}{25}, t_{5}=\frac{35}{18}, \cdots$. Hence $H E(\alpha, k) \backslash H E(\alpha, k+1)=\left(\lambda_{k+1}, \lambda_{k}\right]$, where $\lambda_{1}=\sqrt{\frac{2}{3}}, \lambda_{2}=\frac{3}{4}, \quad \lambda_{3}=$ $\sqrt{\frac{8}{15}}, \lambda_{4}=\sqrt{\frac{25}{48}}, \lambda_{5}=\sqrt{\frac{18}{35}}, \cdots$, and $\operatorname{HE}(\alpha, \infty)=\left(0, \sqrt{\frac{1}{2}}\right]$.

Let $W_{\alpha}$ be a weighted shift whose restriction to $\vee\left\{e_{1}, e_{2}, \cdots\right\}$ is subnormal, with associated Berger measure $\mu$. Then it follows from [Cu1, Proposition 8] that $W_{\alpha}$ is subnormal iff

$$
\frac{1}{t} \in L^{1}(\mu) \quad \text { and } \quad \alpha_{0}^{2} \cdot\left\|\frac{1}{t}\right\|_{L^{1}(\mu)} \leq 1 .
$$

In particular, $W_{\alpha}$ is never subnormal when $\mu(\{0\})>0$. The following example is useful when considering the behavior of Bergman shift extensions. 
Example 3.2. Let

$$
\alpha\left(x_{1}, \cdots, x_{n}\right): x_{n}, \cdots, x_{1}, \sqrt{\frac{m}{m+1}}, \cdots, \sqrt{\frac{m+k-1}{m+k}}, \cdots .
$$

(i) If $1 \leq n \leq m-1$, then

$$
\begin{aligned}
& H E(\alpha, \infty)=\left\{\left(x_{1}, \cdots, x_{n}\right) \mid W_{\alpha\left(x_{1}, \cdots, x_{n}\right)} \text { is subnormal }\right\} \\
& \quad=\left\{\left(\sqrt{\frac{m-1}{m}}, \sqrt{\frac{m-2}{m-1}}, \cdots, \sqrt{\frac{m-n+1}{m-n+2}}, x_{n}\right) \mid 0<x_{n} \leq \sqrt{\frac{m-n}{m-n+1}}\right\} .
\end{aligned}
$$

(ii) If $n \geq m$, then $H E(\alpha, \infty)=\emptyset$.

Proof. (i) First we will find the range of $x_{1}$ needed for the subnormality of $W_{\alpha\left(x_{1}\right)}$. Let $\mu_{1}$ be the probability measure corresponding to the subnormal weighted shift with the weights $\sqrt{\frac{m}{m+1}}, \sqrt{\frac{m+1}{m+2}}, \cdots, \sqrt{\frac{m+k-1}{m+k}}, \cdots$. Then

$$
\int_{[0,1]} t^{k} d \mu_{1}(t)=\frac{m}{m+1} \cdots \frac{m+k-1}{m+k}=\frac{m}{m+k}=\int_{0}^{1} m t^{k+m-1} d t .
$$

So $d \mu_{1}=m t^{m-1} d t$. Since $\|1 / t\|_{L^{1}\left(\mu_{1}\right)}=\frac{m}{m-1}$, by (3.1) we have $x_{1} \leq \sqrt{\frac{m-1}{m}}$. Let $\mu_{2}$ be the probability measure corresponding to the weighted shift with the weights $x_{1}, \sqrt{\frac{m}{m+1}}, \cdots, \sqrt{\frac{m+k-1}{m+k}}, \cdots$. Since

$$
x_{1}^{2} \cdot \frac{m}{m+1} \cdots \frac{m+k-2}{m+k-1}=\int_{0}^{1} t^{k} d \mu_{2},
$$

using a method similar to that described above, we have $d \mu_{2}=x_{1}^{2} m t^{m-2} d t$. Furthermore, since $\mu_{2}[0,1]=1$, we have $x_{1}=\sqrt{\frac{m-1}{m}}$. Hence $d \mu_{2}=(m-1) t^{m-2} d t$. In general, let $\mu_{i}$ be the probability measure corresponding to the weighted shift with the weights $x_{i-1}, \cdots, x_{1}, \sqrt{\frac{m}{m+1}}, \cdots, \sqrt{\frac{m+k-1}{m+k}}, \cdots$. Then it follows easily from mathematical induction that $d \mu_{i}=(m-i+1) t^{m-i} d t, x_{i}=\sqrt{\frac{m-i}{m-i+1}}(1 \leq i \leq n-1)$ and $x_{n} \leq \sqrt{\frac{m-n}{m-n+1}}$.

(ii) Let $\mu_{n}$ be the probability measure corresponding to the weighted shift with the weights $x_{n-1}, \cdots, x_{1}, \sqrt{\frac{m}{m+1}}, \cdots, \sqrt{\frac{m+k-1}{m+k}}, \cdots$. Then

$$
d \mu_{n}=(m-n+1) t^{m-n} d t .
$$

Since $n \geq m$,

$$
\int_{0}^{1} \frac{1}{t} d \mu_{n}=(m-n+1) \cdot \int_{0}^{1} t^{m-n-1} d t=\infty,
$$

which implies that $\frac{1}{t} \notin L^{1}\left(\mu_{n}\right)$. Hence $H E(\alpha, \infty)=\emptyset$.

Example 3.3. Let $W_{\alpha}$ be the weighted shift whose weight sequence is given by $\alpha_{n}:=\sqrt{\frac{n+1}{n+2}}(n \geq 0)$. By (ii) of Example 3.2, $W_{\alpha(x)}$ is not subnormal for any $x>0$. By Theorem 2.1, we have $t_{1}=2, t_{2}=3, t_{3}=\frac{11}{3}, t_{4}=\frac{25}{6}, t_{5}=\frac{137}{30}, \cdots$. Hence $H E(\alpha, k) \backslash H E(\alpha, k+1)=\left(\lambda_{k+1}, \lambda_{k}\right]$, where $\lambda_{1}=\sqrt{\frac{1}{2}}, \lambda_{2}=\sqrt{\frac{1}{3}}, \lambda_{3}=$ $\sqrt{\frac{3}{11}}, \lambda_{4}=\sqrt{\frac{6}{25}}, \lambda_{5}=\sqrt{\frac{30}{137}}, \cdots$, and $H E(\alpha, \infty)=\emptyset$. 
In addition, we consider an example of non-Bergman shift type.

Example 3.4. Let $W_{\alpha}$ be the weighted shift whose weight sequence is given by

$$
\alpha_{n}:=\sqrt{\frac{n+1}{n+2} \cdot \frac{1}{2} \cdot \frac{2^{n+2}-1}{2^{n+1}-1}} \quad(n \geq 0) .
$$

Let $d \mu:=2 \chi_{\left[\frac{1}{2}, 1\right]} d t$. Since $\gamma_{n}=2 \int_{\frac{1}{2}}^{1} t^{n} d t=\frac{1}{n+1} \cdot \frac{1}{2^{n}} \cdot\left(2^{n+1}-1\right), \mu$ is the probability measure corresponding to the weighted shift with a weight sequence $\alpha:=\left\{\alpha_{n}\right\}_{n=0}^{\infty}$. Hence $W_{\alpha}$ is subnormal and, by $(3.1), W_{\alpha(x)}$ is subnormal if and only if $x^{2} \int_{0}^{1} \frac{1}{t} d \mu \leq$ 1 , which is equivalent to $0<x \leq \frac{1}{\sqrt{2 \ln 2}}$. By Theorem 2.1, we have $t_{1}=\frac{4}{3}, t_{2}=$ $\frac{18}{13}, t_{3}=\frac{262}{189}, t_{4}=\frac{445}{321}, t_{5}=\frac{34997}{25245}, \cdots$, and $H E(\alpha, k) \backslash H E(\alpha, k+1)=\left(\lambda_{k+1}, \lambda_{k}\right]$, where $\lambda_{1}=\sqrt{\frac{3}{4}} \approx 0.866025, \lambda_{2}=\sqrt{\frac{13}{18}} \approx 0.849837, \lambda_{3}=\sqrt{\frac{189}{262}} \approx 0.849337, \lambda_{4}=$ $\sqrt{\frac{321}{445}} \approx 0.849322, \lambda_{5}=\sqrt{\frac{25245}{34997}} \approx 0.849322, \cdots$, and $H E(\alpha, \infty)=\left(0, \frac{1}{\sqrt{2 \ln 2}}\right]$, with $\frac{1}{\sqrt{2 \ln 2}} \approx 0.849322$.

3.2. Recursively generated type. Using Proposition 2.4, we can recapture a well-known result (cf. [CuF1, $\mathrm{CuF} 2$ or $\mathrm{CuL}$ ).

Example 3.5. We consider $\alpha:(a, b, c)^{\wedge}$, where $0<a<b<c$. Since $\operatorname{rank} \gamma=2$, by (2.7) $t_{1}=\frac{1}{a^{2}}$ and $t_{2}=\frac{a^{4}-2 a^{2} b^{2}+b^{2} c^{2}}{a^{2} b^{2}\left(c^{2}-b^{2}\right)}$. Hence

(i) $H E(\alpha, 1)=\{x: 0<x \leq a\}$,

(ii) $H E(\alpha, 2)=\cdots=H E(\alpha, \infty)=\left\{x: 0<x \leq a b \sqrt{\frac{c^{2}-b^{2}}{a^{4}-2 a^{2} b^{2}+b^{2} c^{2}}}\right\}$.

We close the paper with the following example.

Example 3.6. Let $\alpha:(a, b, c, d, e)^{\wedge}$ with $0<a<b<c<d<e$ satisfying

$$
\frac{b^{2}}{c^{2}} \cdot \frac{c^{4}-2 a^{2} c^{2}+a^{2} b^{2}}{b^{2}-a^{2}} \leq d^{2} \quad \text { and } \quad \frac{c^{2}}{d^{2}} \cdot \frac{d^{4}-2 b^{2} d^{2}+b^{2} c^{2}}{c^{2}-b^{2}} \leq e^{2} .
$$

Then by [Li Corollary 2.12], $W_{\alpha}$ is subnormal. Assume that rank $\gamma=3$. Then by (1.1),

$$
\alpha_{n}^{2}=\psi_{2}+\frac{\psi_{1}}{\alpha_{n-1}^{2}}+\frac{\psi_{0}}{\alpha_{n-1}^{2} \cdot \alpha_{n-2}^{2}} \quad(n \geq 3)
$$

with

$$
\begin{aligned}
& \psi_{0}=\frac{a^{2} b^{2} c^{2}\left(b^{2} c^{4}-2 b^{2} c^{2} d^{2}+c^{2} d^{4}+b^{2} d^{2} e^{2}-c^{2} d^{2} e^{2}\right)}{a^{2} b^{4}-2 a^{2} b^{2} c^{2}+b^{2} c^{4}+a^{2} c^{2} d^{2}-b^{2} c^{2} d^{2}} \\
& \psi_{1}=-\frac{b^{2} c^{2}\left(a^{2} b^{2} c^{2}-a^{2} b^{2} d^{2}-a^{2} c^{2} d^{2}+c^{2} d^{4}+a^{2} d^{2} e^{2}-c^{2} d^{2} e^{2}\right)}{a^{2} b^{4}-2 a^{2} b^{2} c^{2}+b^{2} c^{4}+a^{2} c^{2} d^{2}-b^{2} c^{2} d^{2}} \\
& \psi_{2}=\frac{c^{2}\left(a^{2} b^{4}-a^{2} b^{2} c^{2}-a^{2} b^{2} d^{2}+b^{2} c^{2} d^{2}+a^{2} d^{2} e^{2}-b^{2} d^{2} e^{2}\right)}{a^{2} b^{4}-2 a^{2} b^{2} c^{2}+b^{2} c^{4}+a^{2} c^{2} d^{2}-b^{2} c^{2} d^{2}}
\end{aligned}
$$

Hence by (2.7), $t_{1}=\frac{1}{a^{2}}, \quad t_{2}=\frac{a^{4}-2 a^{2} b^{2}+b^{2} c^{2}}{a^{2} b^{2}\left(c^{2}-b^{2}\right)}$, and $t_{3}=\frac{A}{B}$, where

$$
\begin{aligned}
A= & a^{4} b^{6}-3 a^{4} b^{4} c^{2}+a^{4} b^{2} c^{4}+2 a^{2} b^{4} c^{4}+2 a^{4} b^{2} c^{2} d^{2}-2 a^{2} b^{4} c^{2} d^{2}-2 a^{2} b^{2} c^{4} d^{2} \\
& +b^{2} c^{4} d^{4}-a^{4} c^{2} d^{2} e^{2}+2 a^{2} b^{2} c^{2} d^{2} e^{2}-b^{2} c^{4} d^{2} e^{2} \\
B= & a^{2} b^{2} c^{2}\left(b^{2} c^{4}-2 b^{2} c^{2} d^{2}+c^{2} d^{4}+b^{2} d^{2} e^{2}-c^{2} d^{2} e^{2}\right) .
\end{aligned}
$$


Hence

(i) $H E(\alpha, 1)=\{x: 0<x \leq a\}$,

(ii) $H E(\alpha, 2)=\left\{x: 0<x \leq a b \sqrt{\frac{c^{2}-b^{2}}{a^{4}-2 a^{2} b^{2}+b^{2} c^{2}}}\right\}$,

(iii) $H E(\alpha, 3)=\cdots=H E(\alpha, \infty)=\left\{x: 0<x \leq \sqrt{\frac{B}{A}}\right\}$.

\section{REFERENCES}

[Br] J. Bram, Subnormal operators, Duke Math. J. 22 (1955), 75-94. MR 16:835a

[Cu1] R. Curto, Quadratically hyponormal weighted shifts, Integral Equations and Operator Theory 13 (1990), 49-66. MR 90k:47061

[Cu2] R. Curto, Joint hyponormality: A bridge between hyponormality and subnormality, Proc. Symposia Pure Math. 51 (1990), 69-91. MR 91k:47049

[CuF1] R. Curto and L. Fialkow, Recursively generated weighted shifts and the subnormal completion problem, Integral Equations and Operator Theory 17 (1993), 202-246. MR 94h:47050

[CuF2] R. Curto and L. Fialkow, Recursively generated weighted shifts and the subnormal completion problem, II, Integral Equations and Operator Theory 18 (1994), 369-426. MR 94m:47044

[CuF3] R. Curto and L. Fialkow, Recursiveness, positivity, and truncated moment problems, Houston J. Mathematics 17 (1991), 603-635. MR 93a:47016

[CuJ1] R. Curto and I. Jung, Quadratically hyponormal weighted shifts with first two equal weights, Integral Equations and Operator Theory 37 (2000), 208-231.

[CuL] R. Curto and W. Lee, Joint hyponormality of Toeplitz pairs, Memoirs Amer. Math. Soc., to appear.

[CuP1] R. Curto and M. Putinar, Existence of non-subnormal polynomially hyponormal operators, Bull. Amer. Math. Soc. 25 (1991), 373-378. MR 93e:47028

[CuP2] R. Curto and M. Putinar, Nearly subnormal operators and moment problems, J. Functional Analysis 115 (1993), 480-497. MR 95d:47024

[Gan] F. R. Gantmacher, The theory of matrices, Vol. 1, Chelsea Publ. Co., 1960. MR 21:6372c

[Hal] P. Halmos, Normal dilations and extensions of operators, Summer Bras. Math. 2 (1950), 124-134. MR 13:359b

[Li] X. Li, Moment sequences and their applications, Dissertation, Virginia Polytechnic Institute and State University, 1994.

[ShT] J. A. Shohat and J. D. Tarmakin, The Problem of Moments, Math. Surveys I, American Math. Soc., Providence, 1943. MR 55:5c

[Sta] J. Stampfli, Which weighted shifts are subnormal, Pacific J. Math. 17 (1966), 367-379. MR 33:1740

[Wol] Stephen Wolfram, The Mathematica Book, 3rd ed., Wolfram Media/Cambridge University Press, 1996. MR 97d:68001

Department of Mathematics, Kyungpook National University, Taegu 702-701, Korea

E-mail address: ibjung@kyungpook.ac.kr

Department of Mathematics, Yanbian University, Yanji 133-002, People's Republic OF ChINA

Current address: TGRC, Kyungpook National University, Taegu 702-701, Korea

E-mail address: chunjili@hanmail.com 